\title{
Should Fear of Adverse Events Influence the Decision to Administer Preoperative Therapy to Patients with Pancreatic Cancer?
}

\author{
Matthew H. G. Katz \\ Pancreatic Surgical Service, Department of Surgical Oncology, The University of Texas MD Anderson Cancer Center, \\ Houston, TX
}

For almost 30 years, oncologists have debated the rationale for delivering chemotherapy, radiation therapy, or both to patients anticipated to undergo pancreatectomy for localized adenocarcinoma. This issue of Annals of Surgical Oncology reports a study by Dr. Marchegiani and colleagues that retrospectively evaluated clinical data from patients who underwent resection for pancreatic cancer at their institution. The study found that patients who received chemotherapy before pancreatoduodenectomy experienced postoperative adverse events at a rate similar to that of patients who did not receive it. However, the adverse events experienced by the patients who received preoperative chemotherapy were associated with an increased clinical burden. ${ }^{1}$ The authors seem to interpret their data as supporting the performance of pancreatectomy de novo whenever technically possible to minimize perioperative adverse events. But, is that the correct interpretation?

The question is not an easy one to answer. The clinical excellence of the authors' group notwithstanding, the data they provide are limited in scope, and their conclusions appear, at least to some extent, clouded by bias against the administration of preoperative therapy to patients with resectable tumors. Indeed, only one-third of the patients in this series were treated with chemotherapy before pancreatectomy. Moreover, although their disease stages are not reported, it appears that these patients generally had cancers that were anatomically more advanced than those of

(C) Society of Surgical Oncology 2017

First Received: 26 November 2017; Published Online: 14 December 2017

M. H. G. Katz

e-mail:Mhgkatz@mdanderson.org patients who underwent primary resection. Their operations were longer, involved more blood loss, and required concomitant vascular resection and reconstruction more frequently.

Whether surgery after the administration of preoperative therapy is more technically demanding as a direct result of the pretreatment, as suggested by the authors, or whether it simply appeared so to the authors as the result of their preferential administration of preoperative therapy to patients who had tumors that were inherently technically more demanding to remove is therefore unclear. Similarly, the extent to which the increased clinical burden of perioperative complications observed in association with preoperative therapy was an unfavorable result of the cytotoxic treatments themselves or simply an artifact of their development after surgical procedures significantly more complex also is uncertain.

Despite this apparent treatment bias, Dr. Marchegiani and colleagues found no differences in rates of overall morbidity, Clavien-Dindo severity ranking, or perioperative mortality between patients treated with preoperative therapy and those who underwent primary resection. Furthermore, preoperative therapy was associated with a reduced rate of pancreatic fistula, pancreatitis, and hemorrhage among the patients who underwent pancreatoduodenectomy.

These encouraging findings are similar to ours and those of others clearly demonstrating that preoperative therapyeven radiation therapy-does not increase the incidence of surgical complications and that its administration should not be avoided on this basis., ${ }^{2,3}$ On the contrary, given the low rate at which patients receive requisite nonoperative therapies after surgery overall, and given that postoperative complications may further limit the ability to deliver these 
therapies in the postoperative period, ${ }^{4}$ the delivery of chemotherapy, radiation therapy, or both before surgery has real appeal. Indeed, we previously showed that major perioperative complications are associated with a shorter survival for patients treated primarily with surgery, but that no such adverse association exists among patients treated preoperatively. ${ }^{5}$ We could not determine whether the association was related to the long-term sequelae of inflammation and immunosuppression or merely reflected the frequent inability of patients to receive intended therapy after surgery. Regardless, taken together with the results from this and other prior studies, the data from Dr. Marchegiani's report could be interpreted as additional evidence that the administration of chemotherapy, radiation therapy, or both is safe and highly rational in the preoperative setting.

The administration of preoperative therapy has other purported advantages. Within the framework of this discussion of perioperative morbidity, induction therapy may be viewed as an effective selector for operations associated with overall rates of adverse events as high as $75 \%$, even at expert centers, ${ }^{6}$ and a national overall 90 -day mortality rate of $7.4 \%{ }^{7}$ Furthermore, preoperative chemotherapy, radiation therapy, or both may lead to a higher rate of microscopically negative (R0) resection, ${ }^{8}$ lower rates of lymphatic metastasis ${ }^{9}$ and local recurrence, ${ }^{10}$ and a longer life span, ${ }^{8}$ even in the setting of resectable disease. Additional, compelling data derived largely from both preclinical models and single-arm phase 2 trials suggest that at least some patients benefit from a treatment strategy that is not, at least initially, surgical.

Nonetheless, the role of preoperative therapy continues to be debated because no randomized trial evaluating its efficacy has been completed successfully to date. It could easily be argued, however, that we in the United States are already moving forward without such a trial. On the basis of existing evidence, National Comprehensive Cancer Network guidelines ${ }^{11}$ recommend the administration of preoperative therapy to all patients with borderline resectable tumors, and American Society for Clinical Oncology guidelines ${ }^{12}$ acknowledge that its administration is acceptable and appropriate even for patients with resectable disease. Moreover, the only two trials for patients with localized pancreatic cancer currently offered through the National Cancer Institute's National Clinical Trials Network randomize patients to one of two preoperative treatment regimens. Neither trial offers an arm in which patients are treated primarily with pancreatectomy. ${ }^{13,14}$

Like data from most studies on the subject of treatment sequencing for resectable and borderline resectable pancreatic cancer, the data presented by Dr. Marchegiani are equally as unlikely to persuade oncologists to offer routine treatment with surgery de novo as they are to persuade them to administer preoperative therapy routinely, and they should not. Indeed, treatment sequencing decisions should be individualized and based not solely on the anatomic relationships of a single tumor within the pancreas, but also on the estimated extent of the radiographically occult systemic cancer that exists in virtually all patients and the physiologic profile of the specific host within which it develops. Even at the University of Texas MD Anderson Cancer Center, where we have used chemotherapy, radiation therapy, or both to treat patients with localized pancreatic cancer in anticipation of pancreatectomy for the last quarter century, ${ }^{15}$ approximately $20 \%$ of all pancreatic resections for cancer are performed de novo. In general, the strategy is reserved for physiologically robust patients whose cancers appear both anatomically and biologically favorable, and who are anticipated to have a low risk for perioperative adverse events and a high probability of rapid recovery after surgery so that the potential for postoperative therapy is maximized. In contrast, we favor the administration of preoperative therapy to the majority of patients who present with disease that appears less favorable or for whom the delivery of postoperative therapy is less likely. Overall, the results of the study by Dr. Marchegiani and colleagues do not appear inconsistent with this approach.

\section{REFERENCES}

1. Marchegiani, G. Neoadjuvant therapy versus upfront resection for pancreatic cancer: the actual spectrum and clinical burden of post-operative complications. Ann Surg Oncol. 2017. https://doi. org/10.1245/s10434-017-6281-9.

2. Denbo JW, Bruno ML, Cloyd JM, et al. Preoperative chemoradiation for pancreatic adenocarcinoma does not increase 90-day postoperative morbidity or mortality. J Gastrointest Surg. 2016;20:1975-85.

3. Cooper AB, Parmar AD, Riall TS, et al. Does the use of neoadjuvant therapy for pancreatic adenocarcinoma increase postoperative morbidity and mortality rates? J Gastrointest Surg. 2015;19:80-6.

4. Merkow RP, et al. Postoperative complications reduce adjuvant chemotherapy use in resectable pancreatic cancer. Ann Surg. 2014;260:372-7.

5. Tzeng CW, et al. Treatment sequencing for resectable pancreatic cancer: influence of early metastases and surgical complications on multimodality therapy completion and survival. J Gastrointest Surg. 2014;18:16-24. discussion 24-15.

6. Schwarz L, Bruno M, Parker NH, et al. Active surveillance for adverse events within 90 days: the standard for reporting surgical outcomes after pancreatectomy. Ann Surg Oncol. 2015;22:3522-9.

7. Swanson RS, Pezzi CM, Mallin K, Loomis AM, Winchester DP. The 90-day mortality after pancreatectomy for cancer is double the 30-day mortality: more than 20,000 resections from the national cancer data base. Ann Surg Oncol. 2014;21:4059-67.

8. Mokdad AA, Minter RM, Zhu H, et al. Neoadjuvant therapy followed by resection versus upfront resection for 
resectable pancreaticcancer: a propensity score-matched analysis. $J$ Clin Oncol. 2016;pii:JCO685081.

9. Roland CL, Yang AD, Katz M, et al. Neoadjuvant therapy is associated with a reduced lymph node ratio in patients with potentially resectable pancreatic cancer. Ann Surg Oncol. 2015;22:1168-75.

10. Katz MH, Wang H, Balachandran A et al. Effect of neoadjuvant chemoradiation and surgical technique on recurrence of localized pancreatic cancer. J Gastrointest Surg. 2012;16:68-78.

11. Tempero MA, Malafa MP, Al-Hawary M et al. Pancreatic adenocarcinoma, version 2.2017, NCCN clinical practice guidelines in oncology. J Natl Compr Canc Netw. 2017;15:1028-61.

12. Khorana AA, et al. Potentially curable pancreatic cancer: American society of clinical oncology clinical practice guideline. J Clin Oncol. 2016;34:2541-56.
13. ClinicalTrials.gov. Combination chemotherapy with or without hypofractionated radiation therapy before surgery in treating patients with pancreatic cancer. https://clinicaltrials.gov/ct2/ show/NCT02839343. Accessed 12 Jan 2017.

14. ClinicalTrials.gov. Combination chemotherapy or gemcitabine hydrochloride and paclitaxel albumin-stabilized nanoparticle formulation before surgery in treating patients with pancreatic cancer that can be removed by surgery. https://clinicaltrials.gov/ ct2/show/NCT02562716. Accessed 12 Jan 2017.

15. Cloyd JM, Katz MHG, Prakash L, et al. Preoperative therapy and pancreatoduodenectomy for pancreatic ductal adenocarcinoma: a 25-year single-institution experience. $J$ Gastrointest Surg. 2017;21:164-74. 